\title{
Robótica Educacional para o ensino de gráficos em cinemática: possibilidade de suporte à necessidade de autonomia a partir de relatos de estudantes do primeiro ano do ensino médio
}

\author{
Toni Fernando Mendes dos Santos ${ }^{1}$ \\ Paulo José Sena dos Santos ${ }^{2}$
}

\author{
${ }^{1}$ Escola Estadual Básica Laércio Caldeira de Andrada - sgttoni@gmail.com \\ ${ }^{2}$ Departamento de Física UFSC - paulo.sena@ufsc.br
}

\section{Educational Robotics for teaching graphics in kinematics: possibility of supporting autonomy based on reports from first-year high school students}

\begin{abstract}
Resumo: Diversas pesquisas têm discutido as dificuldades de aprendizagem e a falta de interesse dos estudantes na disciplina de Física. Com o objetivo de minimizar tais problemas alguns autores sinalizam a possibilidade do uso das novas tecnologias, como a Robótica Educacional, que tem recebido destaque nos últimos anos. Alguns trabalhos apontam que o uso da ferramenta pode melhorar a motivação e contribuir com a aprendizagem dos estudantes. Baseados na teoria da autodeterminação, investigamos se as atividades com robótica para o ensino de gráficos em cinemática são capazes de criar contextos com suporte à necessidade psicológica básica de autonomia. A partir de entrevistas semiestruturadas com 13 estudantes concluímos que os contextos gerados pela robótica permitiram perceber a existência de escolhas e momentos de autoria, condições importantes para a promoção da autonomia.
\end{abstract}

Palavras-chave: Robótica Educacional, suporte à autonomia, cinemática, teoria da autodeterminação

\begin{abstract}
Several researches have discussed learning difficulties and students' lack of interest in Physics. In order to minimize such problems, some authors point to the possibility of using new technologies, such as educational robotics, which has been highlighted in recent years. Some studies indicate that the use of this tool can improve students' motivation. Thus, based on the theory of self-determination, we investigate whether activities with the use of robotics for teaching graphics in kinematics are capable of creating contexts that support the basic psychological need for autonomy. From semi-structured interviews with 13 students, we concluded that the contexts generated by robotics allowed us to perceive the existence of choices and moments of authorship, important conditions for the promotion of autonomy.
\end{abstract}

Keywords: Educational Robotics, autonomy support, kinematics, self-determination theory

\section{Introdução}

Há décadas trabalhos têm discutido as dificuldades de aprendizagem dos alunos na componente curricular Física. Em geral, tais dificuldades estão, boa parte, associadas a aspectos como: a grande idealização, o que não permite uma associação direta entre os conceitos discutidos em sala com o cotidiano; a forma como os conteúdos são abordados, excessivamente presos à matematização, com abordagens centradas na resolução de exercícios; e a pouca contextualização. Assim, o ensino de Física se apresenta distante, não conseguindo despertar a curiosidade nem o interesse dos alunos, elementos importantes da motivação para aprender (CLEMENT, 2013). 
Geralmente o primeiro contato dos estudantes com a Física, seja no nono ano do fundamental ou no primeiro ano do médio, é através do estudo da cinemática. Entre os diversos assuntos discutidos, um dos que mais recebe atenção dos pesquisadores diz respeito ao uso da representação gráfica, pois o uso da linguagem gráfica permite a síntese de informações e o estabelecimento de relações entre diferentes grandezas não só na Física, mas em diversas outras áreas do conhecimento (BEICHNER, 1994; IVANJEK et al, 2016).

Com objetivo de melhorar a aprendizagem e aumentar o interesse dos estudantes, alguns autores defendem o uso de atividades experimentais e das novas tecnologias, em particular, o uso da Robótica Educacional, a qual apresenta potencial para contribuir com a proposição de contextos diferenciados, capazes de fomentar a discussão de diferentes conceitos físicos. Em relação ao ensino de cinemática, nos últimos anos o uso desse recurso tem sido objeto de investigações (MITNIK et al, 2009; TRENTIN et al, 2015; SIQUEIRA; SANTOS, 2019). Além de evidências para a aprendizagem, trabalhos com robótica sinalizam a possibilidade de melhoraria na qualidade motivacional dos estudantes (FORNAZZA; WEBER, 2014; TEIXEIRA et al., 2018).

Considerando o contexto escolar, diversos pesquisadores têm apontado a motivação como um elemento importante para a aprendizagem (BZUNECK, 2009), pois o rendimento acadêmico não pode ser explicado unicamente por conceitos como inteligência, contexto familiar e condição socioeconômica, a motivação para aprender constitui-se numa variável relevante (LOURENÇO; DE PAIVA, 2010). De forma geral, os estudos sobre motivação se concentram no que direciona e energiza o comportamento humano (RYAN; DECI, 2017), ou seja, têm por foco os motivos que geram determinados comportamentos. E no contexto escolar, o comportamento desejado é o que leva a aprendizagem.

Pelos motivos expostos, faz-se importante a produção de pesquisas que avaliem de forma sistemática que impacto o uso da Robótica Educacional pode ter na qualidade motivacional dos estudantes. Em particular, nosso objetivo é discutir como a robótica pode contribuir para melhorar a motivação para aprender Física. Neste artigo apresentamos os resultados de um estudo qualitativo realizado com o objetivo de identificar como as atividades que desenvolvemos com o uso da robótica para o ensino e a aprendizagem de gráficos em cinemática podem afetar a qualidade motivacional dos estudantes. Para isto, avaliamos, a partir do relato de 13 alunos, se os contextos propiciados foram capazes de dar suporte às suas necessidades psicológicas básicas, condição necessária para a promoção de uma boa qualidade motivacional. Para o estudo da motivação, baseamo-nos na teoria da autodeterminação (RYAN; DECI, 2017). Devido ao volume de dados e a complexidade do tema, teremos como foco a análise da necessidade psicológica básica de autonomia.

\section{As necessidades psicológicas básicas: a autonomia em foco}

A teoria da autodeterminação (RYAN; DECI, 2017) investiga criticamente tanto fatores intrínsecos ao desenvolvimento individual, quanto extrínsecos presentes nos contextos sociais, que podem facilitar a motivação, a integração social e o bem-estar ou, em caso contrário, contribuir para a fragmentação, comportamentos antissociais e infelicidade.

Um dos pressupostos da teoria é que os fatores sociocontextuais podem promover ou dificultar o desenvolvimento dos indivíduos através da satisfação de três necessidades psicológicas básicas: autonomia, competência e pertencimento. Porém, segundo os autores, as próprias necessidades podem variar dentro de uma pessoa ao longo do tempo, assim como dentro de contextos e interações sociais diferentes. 
De forma geral, a necessidade de autonomia envolve experimentar autodeterminação. Trata-se da necessidade que um indivíduo tem de compreender o início e a regulação de um comportamento como seus. O sentimento de autonomia pode surgir quando o indivíduo segue seus interesses, preferências e vontades. No contexto escolar, essa necessidade pode ser potencialmente satisfeita quando, dentre outras coisas, o aluno sentir-se responsável por suas ações e entender que possui certas liberdades de escolha.

Embora autoria e autonomia sejam construtos distintos, entendemos que fornecer mais momentos onde o estudante sinta-se autor de sua aprendizagem pode contribuir para a satisfação da necessidade de autonomia ao facilitar a percepção de liberdade de escolha. Em relação às escolhas que o aluno pode fazer dentro da sala de aula, Stefanou et al. (2004) argumentam que a experiência de autonomia diferencia-se qualitativamente em três tipos: autonomia organizacional, procedimental e cognitiva.

- Organizacional: relaciona-se com a possibilidade de gerenciamento da sala de aula, como o desenvolvimento de regras em forma conjunta, escolha da forma de trabalhar - em grupo ou individual, as formas de avaliação e prazos para entrega de trabalhos;

- Procedimental ou processual: relaciona-se as ações do professor que oferecem aos estudantes a possibilidade de escolha nas formas de apresentação de suas ideias, competências;

- Cognitiva: relaciona-se as ações do professor que incentivam a apropriação de conceitos por parte do aluno, como a solicitação de justificativa ou defesa de suas ideias, a construção de seus próprios caminhos para a solução de problemas e avaliação do processo.

Embora seja ideal oferecer suporte a todas, Stefanou et al. (2004) apontam que a autonomia cognitiva é aquela que se relaciona com a promoção de um investimento psicológico mais duradouro por parte do aluno. Assim, sustentam que o suporte às demais autonomias é importante e necessário, mas não suficiente para que o aluno se engaje profundamente nas atividades.

Em atividades que envolvam o uso da Robótica Educacional, ações como: possibilitar a escolha dos membros do grupo; a organização do espaço de trabalho; a escolha dos materiais para a construção dos modelos a serem prototipados; maior espaço para a discussão de ideias (entre aluno - aluno e aluno - professor); a tomada de decisões; a avaliação de erros; o recebimento de feedback informativo; e a exploração da criatividade para encontrar a solução de um problema apresentam potencial para aumentar o sentimento de autonomia, principalmente quando comparado às aulas tradicionais.

\section{Metodologia}

\subsection{Dos materiais e da organização das atividades}

Os materiais utilizados basearam-se na placa Arduino. As primeiras atividades tiveram como objetivo apresentar alguns componentes eletrônicos e elementos de lógica de programação. Nesse momento, foram utilizados os kits de robótica da Atto Educacional adquiridos pela escola (Figura 1). Os kits possuem sensores (distância, pressão, magnético, luz, temperatura, entre outros) e atuadores (LEDs, motores DC e servo motores) e uma placa (AttoBox) preparada para que diferentes elementos sejam conectados via USB.

$\mathrm{Na}$ sequência das atividades foram utilizados protótipos veiculares (Figura 2), os "carrinhos", planejados e desenvolvidos pelos pesquisadores. O chassi dos carrinhos, rodas e suportes (para motores e roda "boba") foram produzidos em uma impressora 
3D. Estavam acoplados ao chassi a placa Arduino e o motor shield L293D (uma placa que permite que sejam ligados até quatro motores com uso de alimentação externa) e dois motores de corrente contínua.

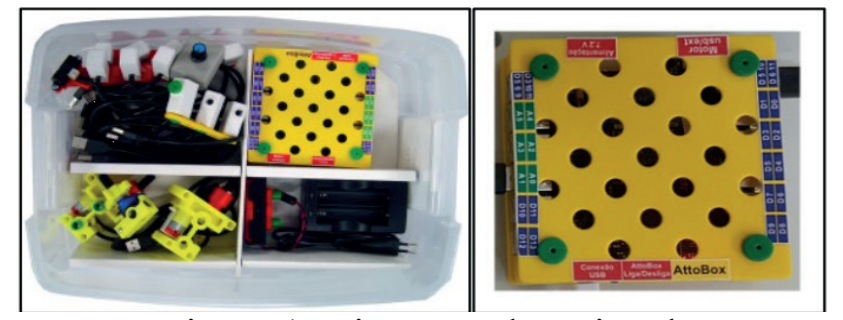

Figura 1. Kit Atto Educacional

Fonte: os autores

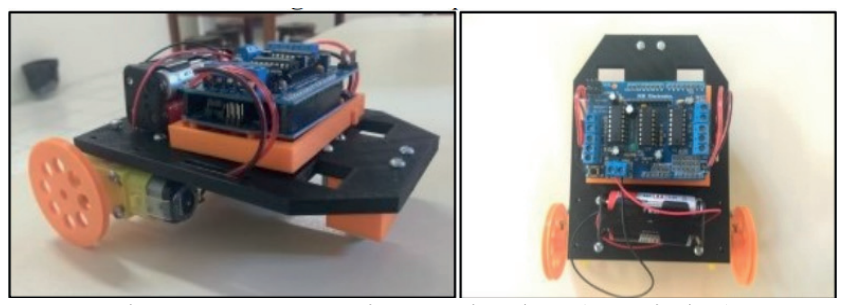

Figura 2. Protótipo veicular (carrinho)

Fonte: os autores

Em todas as atividades a programação foi realizada no Ardublock, programa que funciona de forma associada ao ambiente de programação próprio do Arduino (IDE). $\mathrm{O}$ Ardublock possibilita a programação através de blocos que se encaixam.

Para fins de organização didático-metodológica, as atividades foram desenvolvidas em três fases distintas. Uma fase de apresentação dos materiais ( $1^{\mathrm{a}}$ fase), uma fase de exploração ( $2^{\text {a }}$ fase) e uma fase de aplicação dos conhecimentos trabalhados ( $3^{\mathrm{a}}$ fase $)$ :

- $\quad \mathbf{1}^{\mathrm{a}}$ Fase: planejada para que os alunos pudessem conhecer e explorar os kits de robótica, o ambiente e a linguagem de programação. Nessa fase foram utilizados os kits Atto Educacional, e os alunos realizaram diversas atividades como: acender e apagar led, construir um semáforo, controlar motores, entre outras. Em algumas tarefas a programação foi discutida previamente, enquanto outras foram em formato de desafio. As atividades ocuparam 04 aulas (160 min).

- $\mathbf{2}^{\mathbf{a}}$ fase: essa etapa teve por objetivos relacionados à robótica, a exploração e a consolidação dos elementos de lógica de programação. No ensino de física, teve por finalidade abordar a construção e a interpretação de gráficos. Nessa fase as equipes programaram seus carrinhos para realizar diferentes movimentos (todos retilíneos e uniformes), gravaram vídeos do carrinho em movimento para coletar informações, construíram e analisaram os gráficos produzidos. Essa fase utilizou 08 aulas (320 min).

- $3^{\text {a }}$ fase: na terceira e última parte, os alunos utilizaram o carrinho para discutir o conceito de velocidade escalar média. Foi proposto aos alunos elaborar diferentes formas para obter a velocidade média do carrinho, pois a fornecida na programação não correspondia a velocidade real do movimento. Assim, os grupos deveriam discutir, planejar e implementar suas ideias, apresentando em um relatório o que foi discutido para chegarem às abordagens implementadas e quais resultados foram obtidos. Cada grupo poderia estruturar e apresentar o relatório da forma que desejasse (vídeos, podcasts, blogs ou impresso), desde 
que alguns elementos previamente acordados estivessem presentes. Foram destinadas 04 aulas (160 min) para essa parte.

Além de estruturarmos as atividades em etapas, cada qual com seus objetivos específicos, baseados na teoria da autodeterminação e na proposição de Stefanou et al (2004), procuramos oferecer aos alunos contextos onde tivessem a oportunidade de experienciar diferentes tipos de escolhas (Quadro 1).

Quadro 1: Oportunidades de escolha baseados nos diferentes tipos de autonomia

\begin{tabular}{|l|l|l|}
\hline $\begin{array}{l}\text { Autonomia } \\
\text { organizacional }\end{array}$ & $\begin{array}{l}\text { - Escolher os membros do grupo; } \\
\text { - Organizar o espaço de trabalho. }\end{array}$ \\
\hline $\begin{array}{l}\text { Autonomia } \\
\text { procedimental }\end{array}$ & $\begin{array}{l}\text { - Escolher diferentes funções dentro do grupo durante as atividades; } \\
\text { - Sempre que possível, manipular materiais para a montagem das } \\
\text { atividades. }\end{array}$ \\
\hline $\begin{array}{l}\text { Autonomia } \\
\text { cognitiva }\end{array}$ & $\begin{array}{l}\text { - Justificar suas escolhas, defender ideias, argumentar; } \\
\text { - Construir diferentes caminhos para a resolução de um } \\
\text { problema/desafio. }\end{array}$ \\
\hline
\end{tabular}

Fonte: Os autores

\subsection{Contexto e sujeitos}

A pesquisa foi realizada em uma escola da rede pública federal, localizada na cidade de Florianópolis, Santa Catarina. A referida instituição possui diferentes laboratórios (física, línguas estrangeiras, química, entre outros) e um corpo docente formado, em sua maioria, por mestres e doutores. As turmas onde as atividades foram desenvolvidas são atendidas por um professor efetivo com experiência considerável na área, formado em física, que atualmente faz doutorado em Educação Científica e Tecnológica. Esse contexto é diferente do encontrado na maior parte das escolas públicas da cidade.

A sequência foi ministrada por um dos pesquisadores, porém o professor da turma esteve presente em todos os momentos. Participaram das atividades 97 estudantes de quatro turmas do primeiro ano do ensino médio. Porém, nem todos devolveram os Termos de Assentimento/Consentimento Livre e Esclarecido (TALE/TCLE), seus ou de seus responsáveis. Por esse motivo, a amostra foi reduzida para 78 sujeitos. Na etapa da pesquisa descrita nesse trabalho, foram convidados para as entrevistas semiestruturadas 15 estudantes que apresentaram diferentes tipos de envolvimento para com as atividades. Tal avaliação para compor a amostra a ser entrevistada foi realizada pelos pesquisadores durante a aplicação da sequência didática. Entretanto, dos convidados nem todos compareceram. Assim, ao final do processo, 13 estudantes foram entrevistados.

\subsection{Da entrevista}

As questões foram estruturadas com o objetivo de identificar a percepção do aluno acerca do seu interesse e aprendizado; e avaliar a percepção acerca do suporte às diferentes necessidades psicológicas básicas. Nesse trabalho discutiremos apenas questões relacionadas ao suporte à autonomia. Para a elaboração do roteiro da entrevista, baseamo-nos no elaborado por Clement (2013), utilizando ou adaptando algumas das questões propostas. As entrevistas foram gravadas em áudio, transcritas e, posteriormente, analisadas.

Segundo Duarte (2004), podemos estruturar a análise das entrevistas delineando alguns procedimentos que podem ser utilizados para facilitar sua interpretação mediante o referencial teórico escolhido. Assim, com base na teoria da autodeterminação, definimos alguns eixos temáticos, dentre eles, percepção acerca da necessidade de 
autonomia. A partir desses eixos, novos elementos surgiram, possibilitando a estruturação de outras categorias em torno das quais as falas dos entrevistados foram organizadas e analisadas.

Por questões éticas, objetivando preservar a identidade dos participantes, escolhemos substituir o nome dos estudantes pela letra E seguida de um número, o qual organiza e identifica o sujeito nos diferentes momentos de fala. Assim, os alunos foram numerados de 1 a 13, sendo identificados, portanto, de E1 a E13. Durante as falas, nomes que por ventura os entrevistados citaram foram substituídos por nomes fictícios.

Cabe ressaltar que a realização da pesquisa foi autorizada pelo Comitê de Ética em Pesquisa com Seres Humanos (CEPSH) da UFSC, sob Certificado de Apresentação para Apreciação Ética (CAAE) número 12547119.3.0000.0121.

\section{Resultados e discussão}

Para compreendermos se as diferentes possibilidades de escolha oferecidas ao longo da sequência foram suficientes para dar suporte à necessidade de autonomia, perguntamos, inicialmente, aos alunos:

Durante as atividades o professor dava a liberdade para vocês discutirem, apresentarem ideias, fazerem perguntas?

Como você se sentiu ao ter que justificar suas escolhas, apresentar argumentos, pensar em soluções para os problemas propostos?

Das escolhas que você e seu grupo puderam fazer, como: escolher os membros da equipe, elaborar estratégias e poder discutir com os colegas e professor, escolher e manipular materiais, qual você julga ser a mais importante?

A seguir discutiremos os principais aspectos envolvidos nas respostas fornecidas pelos estudantes e, sempre que possível, apresentaremos alguns trechos representativos.

\subsection{Autonomia organizacional: possibilidade de escolher os membros do grupo}

As falas mostraram a valorização da possibilidade de escolher com quem se vai trabalhar e da participação em uma equipe. Dos 13 estudantes, 06 responderam que a escolha do grupo foi importante e fez diferença durante o andamento das atividades. Nesse contexto, a afinidade foi um elemento de destaque. E relacionada à afinidade está a confiança. Segundo os entrevistados, é importante escolher pessoas com as quais você pode contar, como ilustram os extratos a seguir:

\footnotetext{
Acho que [a escolha] do grupo. Porque se tu escolhe um grupo onde nem todo mundo ajuda, tu não tem como discutir com eles e tirar a dúvida deles ou tirar a sua dúvida. Então, acho que a escolha do grupo é o mais importante assim (E1).

Eu acho que escolher os membros faz um pouco de diferença sim, porque eu escolhi pessoas com quem eu tenho mais intimidade e que eu sei que não iam me abandonar na hora de fazer o trabalho. Acho que faz uma diferença, é sempre bom escolher (E13).
}

Para E6 a escolha das estratégias foi o mais importante. Porém, salientou a importância da escolha do grupo para a ocorrência de discussões e a proposição de estratégias. Ainda afirmou que isso facilitou o bom desenvolvimento das outras etapas, como ilustra a fala: 
[...] A escolha do grupo eu acho que é bem importante, porque a nossa turma é nova, a gente se conheceu esse ano praticamente (...). Então, eu acho que formar o grupo, a gente consegue se relacionar melhor. E eu acho interessante ver o que cada um propõe, só que quando a gente não conhece muito bem as pessoas ou não se relaciona muito bem, às vezes não consegue propor nem desenvolver muito bem as estratégias. A gente fica meio com receio, então, acho que são as duas coisas bem importantes [escolher as estratégias e os membros do grupo], assim, que ajudam bastante no desenvolvimento da atividade, faz fluir (E6).

Cabe destacar que, embora a escolha do grupo esteja vinculada à autonomia organizacional numa primeira análise, sentir-se integrado, ter liberdade para expor ideias, opiniões ou dúvidas pode facilitar a satisfação de outra necessidade psicológica básica, a de pertencimento. Que não será abordada nesse trabalho, mas é importante para a promoção/manutenção de uma boa qualidade motivacional.

\title{
4.2. Autonomia procedimental: possibilidade de escolher e manipular diferentes materiais
}

A possibilidade de escolher e manipular materiais não recebeu muito destaque. Isso pode ter relação com o número de atividades em isso foi oportunizado. Comparadas às demais escolhas, em menos atividades eles puderem escolher os materiais com os quais iriam trabalhar. Desta forma, apenas 02 alunos mencionaram que se tratava de algo importante. Ainda assim, hierarquicamente menos relevante que as demais escolhas que puderam fazer. $\mathrm{O}$ extrato da fala de E7 exemplifica esse ponto:

\begin{abstract}
A mais importante? Acredito que, se eu fosse escolher só uma, seriam as estratégias. E se eu fosse escolher duas, poder manipular os materiais. Porque (...) naquela de descobrir a velocidade, se a gente não tivesse toda aquela liberdade acredito que teria uma dificuldade maior pra tentar desenvolver aquilo ali. (...) E essa liberdade foi muito importante porque dava pra notar o desenvolvimento do pessoal. E a manipulação de materiais, ela também foi muito boa porque dava pra explorar mais outros caminhos (...). Então, a liberdade que ele [professor] deu e a manipulação dos materiais foram muito importantes para o desenvolvimento das atividades. (E7).
\end{abstract}

Outro aspecto relacionado à autonomia procedimental foi a liberdade para escolher e desempenhar diferentes funções (programar, filmar, coletar informações ou fazer os gráficos para a análise do movimento) dentro dos grupos. Contudo, observamos que boa parte dos estudantes permaneceu exercendo a mesma função, seja por se sentir mais confortável, mais confiante ou por solicitação do próprio grupo. Assim, o rodízio das funções aconteceram de forma tímida e em poucos grupos.

\subsection{Autonomia cognitiva: possibilidade de elaborar estratégias, discutir com colegas e professor}

Segundo Stefanou et al (2004) somente os suportes às autonomias organizacional e procedimental não são suficientes para a manutenção do engajamento dos estudantes nas atividades. Segundo os autores sem o suporte a autonomia cognitiva o engajamento seria comprometido. Assim, durante as atividades procuramos oportunizar momentos para discussão (dentro dos grupos e entre os grupos), para elaboração de estratégias e liberdade para testes e avaliações dos erros e acertos dentro do processo. Além desses aspectos, o pesquisador que ministrou a sequência procurou oferecer, sempre que possível, suporte através de feedbacks informativos, e orientações aos alunos de forma individual e coletiva. Dos 13 alunos entrevistados, 06 destacaram 
que poder escolher as estratégias ou discutir com seus colegas e com o professor foi essencial.

\begin{abstract}
Eu acho que a escolha do grupo e a escolha... O desenvolvimento de estratégias, porque são coisas bem importantes. Eu acho que principalmente o desenvolvimento de estratégias, (...). E, podendo escolher a gente continua animado e era algo que às vezes a gente entendesse melhor, algo mais interessante, sabe, que a gente gostasse mais (E6).
\end{abstract}

Acho que discutir com o professor e também elaborar as ideias né, acho que são as mais interessantes de se ter liberdade (E9).

É importante ressaltar que o docente deve encorajar esses diálogos para que os alunos sintam-se a vontade. Ele deve, também, oferecer suporte e incentivar os alunos durante o enfrentamento das dificuldades. Com relação às interações com o professor, todos responderam que tiveram um bom suporte em relação às suas dúvidas e que se relacionaram de forma positiva:

Eu acho que foi boa [a interação], porque tudo o que a gente queria saber e tinha de dúvida a gente perguntava e ele sabia como responder, ajudava a gente. Se não sabia, tentava descobrir junto. Era bem participativo e também ajudava com os materiais (E3).

Também buscamos oportunizar aos alunos maior liberdade para discutirem as estratégias dentro de seus grupos, oferecendo tempos para as tomadas de decisão. Dessa forma, os alunos podiam analisar a atividade, avaliar o que sabiam e quais eram suas dúvidas, dialogar entre si e, posteriormente, chamar o professor (se necessário) para apresentar o que haviam discutido. Esse foi um aspecto bastante citado, como ilustram os extratos:

(...) Na verdade a gente ficou bem mais tempo conversando entre o grupo,
resolvendo o que a gente ia fazer e tentando dar o nosso jeito e só chamando
o professor quando necessário, do que o professor explicando. Ele explicou
bem rapidinho no quadro o que a gente tinha que fazer, para a gente
entender e dai a gente meio que ia do nosso jeito, sabe. (E4).

(...) ele [professor] dava uma liberdade muito grande. (...) Na primeira aula, meu grupo desenvolveu [a estratégia], ele deu bastante tempo pra desenvolver. E na segunda aula a gente foi fazer o experimento. Então, ele deu tempo, bastante espaço e material para os alunos tentarem desenvolver as suas estratégias (E7).

Um dos nossos objetivos era oferecer contextos onde os estudantes pudessem discutir a maior parte do tempo. Isso não significou pouca participação do professor/pesquisador, ao contrário, ele esteve durante todo tempo em contato com os estudantes, oferecendo suporte as suas demandas. Entretanto, procurou intervir após as discussões nos grupos. $\mathrm{E}$ as orientações procuravam levantar reflexões sobre as perguntas feitas, exceto nos casos onde o problema apresentado era de ordem técnica, como em falhas de comunicação entre a placa e o computador, ruptura dos fios dos motores, entre outros. Esse aspecto foi reconhecido, como ilustra o extrato da fala do E6

Acho que a gente teve bastante dúvida, em todas as atividades acho que tinha uma dúvida ali de como fazia. A gente perguntava e era respondido, esclarecido. Claro, não ia falar como que fazia o desafio porque senão ia acabar com a brincadeira, mas acho que todas as dúvidas foram tiradas. A gente conseguiu desenvolver tranquilo depois de a gente ter uma noção (E6). 
Embora não possamos assegurar que todos os participantes tenham experienciado autonomia durante as atividades, a fala dos entrevistados indica que eles perceberam diferentes possibilidades de escolha e momentos de autoria dentro do processo de aprendizagem. Assim, com base na análise dos autorrelatos, foi possível perceber que os contextos gerados pelas atividades foram capazes de oferecer suporte à necessidade psicológica básica de autonomia.

\section{Considerações finais}

De maneira geral, investigações realizadas com o uso da Robótica Educacional em diversas áreas do conhecimento apontam a possibilidade do aumento da motivação dos estudantes durante a realização das atividades. Entretanto, devido à complexidade do tema, consideramos necessárias investigações que utilizem referenciais teóricos sobre o constructo para aprofundar as análises.

A simples presença da robótica em sala pode não trazer resultados esperados. Inicialmente, a introdução de uma novidade pode aumentar a curiosidade dos alunos e trazer uma aparente melhora na motivação. Contudo tais comportamentos se dissipam com o tempo à medida que os alunos se familiarizam com os materiais e com a lógica de programação. Palmer (2005) discutiu sobre o "efeito novidade" nas intervenções em sala de aula argumentando que a introdução de novos elementos ou estilos de ensino podem motivar os estudantes simplesmente em virtude de serem incomuns. Em geral, trabalhos que avaliam o uso da robótica como elemento motivador, constituem-se em atividades de pequena duração (menor ou igual a um mês). Com isso, torna-se bastante razoável considerar o efeito novidade em muitos dos casos. Por esse motivo, realizamos a intervenção num período mais extenso buscando relativizar este efeito.

Após a aplicação da sequência didática, buscamos, a partir da fala dos alunos, evidenciar elementos que pudessem sinalizar se as atividades realizadas foram capazes de gerar contextos com suporte a necessidade psicológica de autonomia dos estudantes, contribuindo para melhorar a motivação para aprender Física, em particular, cinemática. Ao analisarmos as entrevistas, em linhas gerais, percebemos que as atividades geraram contextos capazes de oferecer esse suporte, pois as falas indicaram a percepção de possibilidades de escolha e momentos de autoria, elementos importantes para $\mathrm{o}$ sentimento de autonomia. O relato dos estudantes também permitiu concluir que as atividades oportunizaram trabalho cooperativo, o que possibilita a criação de vínculos e a socialização. Assim, o uso da robótica possibilita novas oportunidades de aprendizagem, sendo capaz de oferecer suporte à necessidade de autonomia.

Porém, cabe ressaltar que diversos são os fatores que devem ser considerados no ambiente de sala de aula quando se procura promover ganhos motivacionais nos estudantes. A introdução da robótica, por si só, não é capaz de mobilizar contextos suficientes para manter, a longo prazo, interesse e motivação de qualidade. Questões como o assunto a ser abordado, a postura do professor e o desenho das atividades pode influenciar o andamento e o envolvimento dos alunos para com as atividades.

Para a continuação do trabalho devemos revisitar a sequência buscando reduzir possíveis dificuldades de programação, bem como desenvolver ou adaptar instrumentos para investigar em larga escala o suporte às necessidades psicológicas básicas em situações didáticas que envolvam Robótica Educacional.

\section{Agradecimentos}

O presente trabalho foi realizado com apoio da Coordenação de Aperfeiçoamento de Pessoal de Nível Superior - Brasil (CAPES). Código de Financiamento 001. 


\section{Referências}

BEICHNER, R. Testing student interpretation of kinematics graphs. Am. J. Phys, 58, $734-742,1994$.

BZUNECK, J. A. A motivação do aluno: aspectos introdutórios. In: BORUCHOVITCH, E; BZUNECK, J. A. (Orgs). A motivação do aluno: contribuições da psicologia contemporânea. 4 ed., p. 9 - 36. Petrópolis: Vozes, 2009.

CLEMENT, Luiz. Autoderteminação e ensino por investigação: construindo elementos para a promoção da autonomia em aulas de física, 2013, $334 \mathrm{f}$. Tese (Doutorado em Educação Científica e Tecnológica) - Programa de Pós-graduação em Educação Científica e Tecnológica, Universidade Federal de Santa Catarina, Florianópolis, 2013.

DUARTE, Rosália. Entrevistas em pesquisas qualitativas. Educar em Revista, Curitiba, n. 24, p. 213-225, July/Dec. 2004.

FORNAZZA, R.; WEBBER; C.G Robótica educacional aplicada à aprendizagem em Física. RENOTE Rev. Novas Tec. Educ., 12, n.1, 2014

IVANJEK, L.; SUSAC, A.; PLANINIC, M.; ANDRASEVIC, A.; MILIN-SIPUS, Z. Student reasoning about graphs in different contexts. Physical Review Physics Educational Research, v. 12, 010106, 2016.

LOURENÇO, A. A., DE PAIVA, M. O. A A motivação escolar e o processo de aprendizagem. Revista Ciências \& Cognição, v. 10, n. 2, p. 132 - 141, 2010.

MITNIK, R.; RECABARREN, M.; NUSSBAUM, M.; SOTO, A. Collaborative robotic instruction: a graph teaching experience. Computers \& Education, 53(2), 330 - 342, 2009.

PALMER, D. A motivational view of constructivist-informed teaching. International Journal of Science Education, v. 21, n. 15, p. 1853 - 1881, 2005.

RYAN, R.; DECI, E. Self-determination theory: basic psychological needs in motivation, development and wellness. New York: The Guilford Press, 2017.

SIQUEIRA, J.; SANTOS, P. J. S. Relato sobre o uso da robótica educacional na discussão de gráficos em cinemática em uma turma do primeiro ano do ensino médio de uma escola pública estadual. RENOTE Rev. Novas Tec. Educ., 17(3), 366 - 375, 2019.

SteFAnOU, C.; PERENCEVICH, K. C.; DiCINTIO, M.; TURNER, J. Supporting autonomy in the classroom: ways teachers encourage student decision making and ownership. Educational Psychologist, n. 39, p. 97 - 110, 2004.

TEIXEIRA, E. S.; MOTA, F. A. X; ALEXANDRIA, A. R.; FRANCISCO, R. V. A. A robótica educacional como ferramenta para o ensino de cinemática. Ensaio Avaliação e Políticas Públicas em Educação, v. 8, n. 1, p. 170 - 197, 2018.

TRENTIN, M. A. S.; ROSA, C. T. W.; ROSA, A. B.; TEIXEIRA, A. C. Free educational robotics in physics teaching: from building the robot to creating a didactical proposal of metacognition orientation. Revista Brasileira de Ensino C\&T, 8(3), 274 $292,2015$. 